\title{
Stimulated Raman spectroscopy of analytes evanescently probed by a silicon nitride photonic integrated waveguide
}

\author{
Haolan Zhao ${ }^{1,2}$, Stéphane Clemmen ${ }^{1,2}$, Ali Raza ${ }^{1,2}$, And Roel Baets ${ }^{1,2}$, \\ ${ }^{1}$ Photonics Research Group, INTEC, Ghent University - imec, Technologiepark-Zwijnaarde 15, 9052 Ghent, Belgium \\ ${ }^{2}$ Center for Nano- and Biophotonics, Ghent University, Technologiepark-Zwijnaarde 15, 9052 Ghent, Belgium \\ *Corresponding author: Roel.Baets@UGent.be \\ Compiled March 15, 2018
}

\begin{abstract}
We report the first demonstration of stimulated Raman spectroscopy enhanced by a nanophotonic integrated circuit. The Raman response of low-concentration dimethyl sulfoxide (DMSO) is evanescently probed via $\mathrm{cm}$-long wire-waveguides. A signal enhancement of close to five orders of magnitude as compared to the case of on-chip spontaneous Raman scattering is demonstrated. This significant enhancement factor allows for the use of $\mathrm{CW}$-lasers with $\mathrm{mW}$-level power and uncooled detectors, and therefore sets the basis of future all-on-a-chip Raman spectrometers suitable for both gas and liquid detection. () 2018 Optical Society of America
\end{abstract}

OCIS codes: (130.0130) Integrated optics; (290.5910) Scattering, stimulated Raman; (130.6010); Sensors (230.7370) Waveguides; (300.6450) Spectroscopy, Raman;

http://dx.doi.org/10.1364/ao.XX.XXXXXX

Raman spectroscopy has found wide popularity in biomedical, physical, chemical and environmental areas due to its capability of identifying molecular fingerprints [1-4]. However, the intrinsic weakness of the Raman scattering process typically requires state-of-the-art high-power laser excitation and deepcooled CCD camera detection that hinders broader lower cost applications of Raman spectroscopy.

Considerable efforts have been devoted to miniaturize Raman systems with the aim of drastically reducing cost and size while enhancing the signal intensity [5]. Waveguide enhancement has been proposed and demonstrated as early as in 1972 [6]. By tightly confining both the excitation and the analyte within an optical waveguide, the Raman signal is hugely enhanced via increased interaction volume. Spontaneous Raman spectroscopy in hollow core photonic crystal fibers has been demonstrated for sub-ppm level gas sensing [7, 8]. However, watt-level pump power and liquid-nitrogen-cooled detectors are typically still needed in these demonstrations. Also, the extension to liquid analytes is not straightforward in the fiber platform $[9,10]$.

Silicon nitride nanophotonics is now a very mature photonic platform providing integrated lasers [11], spectrometers [12] and high-performance spectral functionalities such as filters [13], arrayed-waveguide gratings [14] and Bragg gratings [15]. As a CMOS-compatible technology, silicon nitride photonic integrated circuits are promising for providing a fully integrated Raman sensor in a small footprint and at low cost. The silicon nitride platform not only enables Raman-on-a-chip integration, but it is also suitable for both liquid and gas sensing due to its high refractive index. Spontaneous Raman scattering with evanescent excitations and collections has been demonstrated to detect bulk liquid of isopropylalcohol [16], monolayers [17] and gas in ppb-level thanks to a hypersorbent polymer functionalized waveguide [18]. Unfortunately, the waveguide enhancement remains too low for avoiding the use of deep-cooled detectors in this spontaneous Raman scattering modality, which hurdles the demonstration a fully integrated Raman sensor.

Coherent Raman Scattering (CRS) is another widely utilized phenomenon that is capable of enhancing the Raman signal by many orders of magnitude. CRS is typically implemented using two lasers - a pump laser and a Stokes laser - the difference frequency of which excites molecular vibrations in a resonant way. Both of the CRS implementations, coherent anti-stokes Raman scattering (CARS) and stimulated Raman scattering (SRS), have been extensively applied in vibrational imaging [1]. CARS focuses on the detection of a newly generated light at a blue-shift frequency, while SRS focuses on the detection of relative intensity variations of the injected beams. Although CARS has been demonstrated in liquid fill capillaries [19], SRS is preferable for waveguide Raman sensors due to its automatic phase-matching, unlike CARS that requires challenging dispersion engineering to fulfill the phase-matching condition over a rather wide bandwidth. Besides, in the case of SRS, unlike spontaneous Raman and CARS, the resulting photocurrent signal scales with the amplitude of both the original Stokes field and the scattered field, which by itself is an enhancement mechanism sometimes called self-heterodyned detection[1]. Moreover, SRS is capable of reproducing the spontaneous Raman spectra with linear dependence on concentration. CW SRS has been demonstrated in liquid benzene in 1977 [20] and has recently applied for imaging [21]. The potential of waveguide enhanced SRS has already been demonstrated in hollow core photonic crystal fibers (HCPCF) [22, 23] where SRS signal has been observed from $\mathrm{H}_{2}$ and $\mathrm{CO}_{2}$ with 

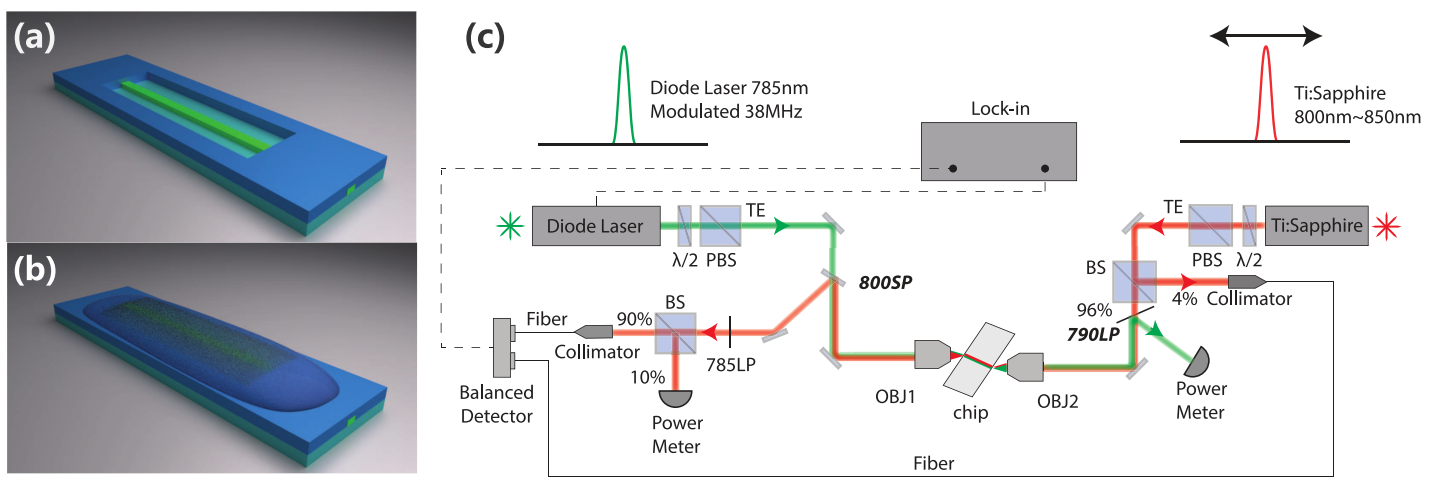

Fig. 1. An illustrative plot of the waveguide (a) before and (b) after the application of DMSO. The cross-section of the waveguide at the sensing section is $h \times w=700 \mathrm{~nm} \times 300 \mathrm{~nm}$. (c) The setup for SRS measurement. We use a counter-propagating configuration with pump coupled from the left to the waveguide and the Stokes injected from the right.

milliwatt level of cw excitations.

Compared to HCPCFs, although evanescent Raman detection with rib waveguides suffers from a reduced signal due to decreased modal overlap, it makes probing of liquids dramatically easier, enables convenient integration with microfluidics[24], and opens up new applications in probing thin-films or even monolayers. On-chip SRS has the potential for on-the-spot detection of 2D materials [25], semiconductor [26], organic material [27], and bio-chemical interactions [17, 28].

In this letter, we report for the first time the use of a nanophotonic waveguide for SRS spectroscopy. As a proof of principle demonstration, the Raman response of dimethyl sulfoxide (DMSO) is recorded from $500 \mathrm{~cm}^{-1}$ to $900 \mathrm{~cm}^{-1}$ with high spectral resolution. Measurements are further performed on the C-S stretch of DMSO at $670 \mathrm{~cm}^{-1}$ to investigate the performance of our setup in low concentration. The detection limit is $280 \mathrm{mM}$ for DMSO with a $600 \mathrm{~ms}$ time constant for the lock-in amplifier and an average power of $60 \mathrm{~mW}$ for both beams. With improvements in light coupling and waveguide design, the sensitivity is expected to be further improved by $3 \sim 4$ orders of magnitudes. Our work paves the way towards a low-cost point-of-care Raman sensor that is expected to find applications in drug production, health monitoring, and on-site environmental sensing.

The Raman sensor is made of a nanophotonic waveguide the geometry of which is chosen as a tradeoff between low propagation loss and high overlap of the evanescent field with the analyte. The silicon nitride waveguide core is deposited through Plasma-Enhanced Chemical Vapor Deposition (PECVD) and later patterned via deep-UV lithography and reactive ion etching as depicted in [16]. The 1-cm long waveguide has a nominal cross-section of $h \times w=700 \mathrm{~nm} \times 300 \mathrm{~nm}$ and its width is tapered up to $3 \mu \mathrm{m}$ at both ends for better coupling efficiency. The bottom optical cladding of the waveguide is a $3.3 \mu \mathrm{m}$-thick layer of silica, and the top cladding is the analyte under investigation along the $8-\mathrm{mm}$ sensing area while the remaining $2-\mathrm{mm}$ is cladded by a layer of $1 \mu \mathrm{m}$-thick silica.

A schematic plot of the structure is shown in Fig. 1(a,b). The refractive index of the silicon nitride is 1.9 , and the mode overlap with the analyte varies as a function of the refractive index of the top-cladding. Pure DMSO has a refractive index of 1.48, resulting in $14 \%$ of the modal power propagating inside the analyte and this power fraction drops to $9.6 \%$ for water. To reduce the impact of reflections at the facet of the waveguide, as discussed later, we terminate the waveguides with a 15-degree angled facet by dicing and polishing of the whole chip.
In the SRS process, the signal appears as a small gain of intensity at the Stokes wavelength and is typically read out via the lock-in technique. In our demonstration, the pump is first amplitude modulated at a certain reference frequency. The modulation is then transferred to the Stokes wave via the Raman interaction and picked up by a lock-in amplifier. By sweeping the Stokes wavelength, we can obtain the Raman spectrum of the analyte. Figure. 1(c) shows the setup of our SRS measurement. A laser diode (LD785-SEV300, Thorlabs) emitting at $785 \mathrm{~nm}$ is used as the pump. It is modulated in intensity with a depth of $30 \%$ at $38 \mathrm{MHz}$ through direct current modulation. A cw tunable Ti: Sapphire laser (SOLSTIS, M2) is employed as the Stokes beam. Two objectives focus pump and Stokes beams from opposite sides of the waveguide. Polarizer and half-wave plates are used to adjust the injected powers and ensure that both beams excite the fundamental TE mode of the waveguide. The transmissions of the beams are monitored for optical alignment. The input power is $30 \mathrm{~mW}$ before the objective for both beams and the time constant of the lock-in amplifier is set to $100 \mathrm{~ms}$ unless explicitly stated. A small part (4\%) of the Stokes beam is tapped before Raman interaction and serves to partially cancel the large DC component in the Raman signal measured by a balanced detector (PDB450A-AC). The electrical signal from the photodiode is then amplified by $10^{4}$ through a built-in transimpedance amplifier.

We first test our setup by the Raman spectrum of the silicon nitride waveguide. For the specific PECVD silicon nitride that makes the core of our waveguide, the Raman response exhibits a sharp peak at $2330 \mathrm{~cm}^{-1}$ and a broad feature below $1200 \mathrm{~cm}^{-1}[16,18]$. We sweep the wavelength from $800 \mathrm{~nm}$ to $850 \mathrm{~nm}$ with a resolution of $0.05 \mathrm{~nm}$ to record the SRS spectrum. It corresponds to a Raman spectrum extending from $295 \mathrm{~cm}^{-1}$ to $1050 \mathrm{~cm}^{-1}$ with $0.74 \mathrm{~cm}^{-1}$ resolution (given the pump wavelength of $785 \mathrm{~nm}$ ). In Fig. 2 we compare the response of the waveguide using spontaneous and stimulated excitation. We can readily see the excellent agreement of both spectra without any adjustment other than a proportionality factor.

To record the SRS spectra of DMSO, we scan the wavelength of the Stokes beam from $817 \mathrm{~nm}$ to $845 \mathrm{~nm}$ with a resolution of $0.05 \mathrm{~nm}$. In Fig. 3 we show the Raman spectra before (dashed red line) and after (solid blue line) drop casting the DMSO. The SRS results are obtained by normalizing the lock-in signal by the intensity of both waves on the chip. After the application of DMSO, we can readily notice the appearance of two peaks at $668.8 \mathrm{~cm}^{-1}$ and $699.3 \mathrm{~cm}^{-1}$ the position and relative intensity of which are in good agreement with the spontaneous Raman spec- 


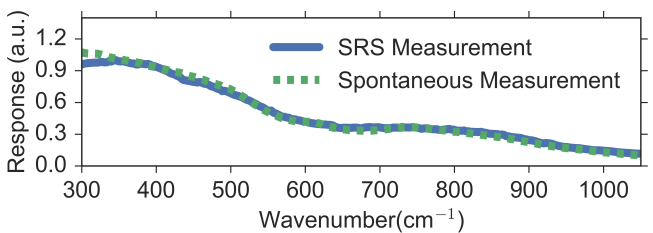

Fig. 2. The response of the waveguide core measured with spontaneous Raman scattering(green dashed) and SRS scattering(blue solid).

tra obtained with a confocal Raman microscope (black dotted line) and with the same waveguide (solid green line).

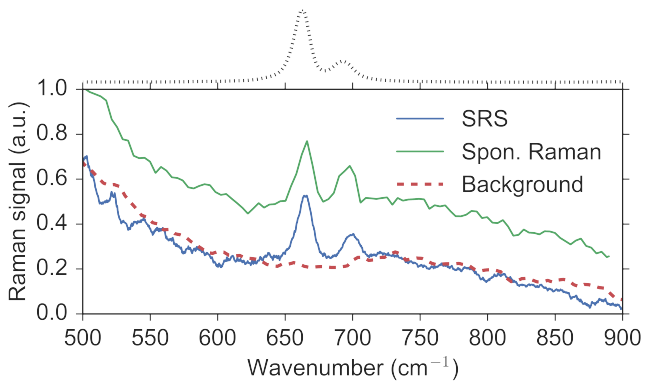

Fig. 3. The recorded SRS spectra from waveguide before (dashed red) and after (solid blue) the application of DMSO. Reference spontaneous Raman spectra for DMSO are taken separately with confocal Raman microscope (black dotted) and with the same waveguide (solid green).

In Fig. 4, the signal dependence on the concentration of DMSO in water is investigated using the $670 \mathrm{~cm}^{-1}$ Raman peak. Fig. 4(a) displays in blue lines the SRS spectra with 50\%, 15\% and $2 \%$ of DMSO dissolved in water recorded with a time constant of $100 \mathrm{~ms}$ and the spectra are vertically offset for clarity. In the $2 \%$ measurement, the SRS spectrum is so noisy that the Raman peak is not identifiable. The Raman peak reappears after we extend the time constant to $600 \mathrm{~ms}$. The result is shown Fig. 4(b) as the red curve and the $100 \mathrm{~ms}$ result is repeated in blue, , and both of the results are subtracted by the mean value. The heights of the DMSO peak relative to the background are extracted, and the results are shown in Fig. 4(c). There is a gradual frequency shift of the Raman peak with reduced concentrations, which might arise from the dipole-dipole interaction of the DMSO-water complex [29]. This frequency shift is also observed in microscope spontaneous Raman measurements. The height of the Raman peaks shows an excellent linearity concerning the DMSO concentration, which is crucial for quantitative analysis. For an input of $60 \mathrm{~mW}$ from both lasers, corresponding to $10 \mathrm{~mW}$ and $3 \mathrm{~mW}$ of effective Stokes and Pump power on the chip, the raw SNR of the $2 \%(280 \mathrm{mM})$ of DMSO is estimated to be 1.5 . The signal level in the lock-in is $44 \mathrm{nV}$, and it corresponds to a modulation depth of $2 \times 10^{-8}$.

The SRS is generally accompanied by two types of parasitic nonlinear processes, namely two-photon absorption and nonlinear phase change $[1,30]$. Two-photon absorption (TPA) refers to the simultaneous absorption of a pump photon and a Stokes photon, and it results in direct attenuation of the Stokes signal. In on-chip SRS, the large bandgap of silicon nitride and the low peak power of the excitations ensure a negligible contribution from TPA. Nonlinear phase change, or cross-phase modulation
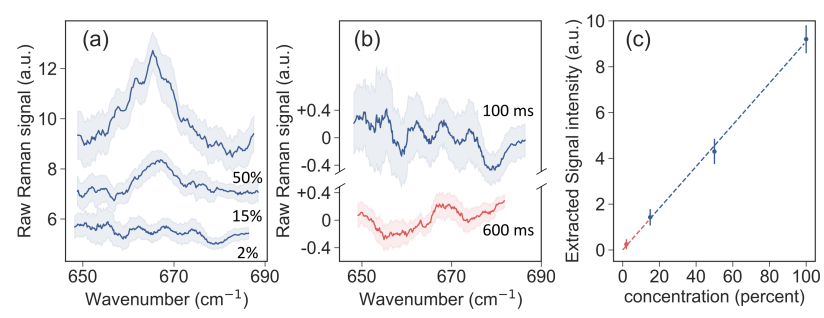

Fig. 4. The SRS signal of different concentrations of DMSO in water. (a) SRS spectra of $50 \%, 15 \%$, and $2 \%$ of DMSO measured with a time constant of $100 \mathrm{~ms}$ (blue) with a y-axis offset for clarity. (b) Mean-value-subtracted SRS spectra of $2 \%$ of DMSO measured with both $100 \mathrm{~ms}$ (blue) and $600 \mathrm{~ms}$ (red). (c) The extracted peak height from the SRS spectra and its standard deviation.

(XPM), arises from the refractive index change induced by the pump beam and experienced by the probe beam. The refractive index change can arise either from thermal effects or from the Kerr effect. Nonlinear phase change per se does not change the amplitude of the Stokes beam and is therefore not expected to provide any spurious signal. However, in practice the nonlinear phase change finds two interferometric paths converting it into amplitude change and the SRS signal can be swamped entirely by them: (i) The silicon nitride waveguide forms intrinsically a low-quality-factor Fabry-Perot cavity; (ii) After the application of the liquid, a small portion of the Stokes beam can reach the other facet directly through the liquid cladding. This spurious transmission and the waveguide transmission constitute an unbalanced Mach-Zehnder interferometer.

In our demonstration, we have suppressed the Fabry-Perot effect by limiting the reflections from the facets through terminating our waveguides with an angle of 15 degrees. This angle reduces by at least $40 \mathrm{~dB}$ the coupling of reflected light back to the waveguide. The Mach-Zehnder effect is circumvented by avoiding injecting both beams on the same line as shown in Fig. 1(c). With these modifications, we were successful in rejecting the spurious signals arising from the XPM and obtain the Raman signal only.

To characterize the signal enhancement provided by the SRS technique, with both the $670 \mathrm{~cm}^{-1}$ and the $700 \mathrm{~cm}^{-1}$ Raman modes, we compare the generated optical power in spontaneous Raman measurement [16] to the power modulation in SRS measurement based on the result in Fig. 3 obtained on the same waveguide. As a result, for $30 \mathrm{~mW}$ of Stokes power before the chip, the experimental signal enhancement is estimated to be $(1.5 \pm 0.8) \times 10^{5}$ with the same pump power. In theory, the enhancement ratio of SRS can be estimated through [1]

$$
\text { Rate }_{\text {SRS }} / \text { Rate }_{\text {Spon }}=n_{S}+1 \text {, }
$$

where $n_{S}$ is the number of Stokes photons within the analyte. We estimate the Stokes power in the analyte to be $0.7 \mathrm{~mW}$ given the estimate of $14 \%$ for the confinement factor. Taking into account the propagation constant and the waveguide length, we estimate the enhancement ratio to be $(1.2 \pm 0.4) \times 10^{5}$, which is in reasonable agreement with the experiments.

Although the stimulation by the Stokes beam dramatically intensifies the Raman signal, it also induces extra shot noise to the system. In our optical setup, the shot noise associated with $1 \mathrm{~mW}$ of Stokes power in the balanced detector (corresponding to the maximum power injection in the measurements) is found 
to be $41 \mathrm{nV} / \sqrt{\mathrm{Hz}}$ while the dark noise is $71 \mathrm{nV} / \sqrt{\mathrm{Hz}}$. This implies that the dark noise still limits the current setup, and an SNR of 1.8 is expected in the $2 \%$ DMSO measurement while the measured value is 1.5. For comparison, the SNR is 1.9 for onchip spontaneous measurement with a $30 \mathrm{~s}$ integration time and the same $3 \mathrm{~mW}$ pump power on the chip. The integration time is chosen to be the same as recording the spectra of $2 \%$ DMSO in Fig. 4(b) with $3 \mathrm{~cm}^{-1}$ resolution. We can see that a truly shotnoise limited SRS system would outperform the spontaneous one regarding SNR despite the extra shot noise.

In the present experiments, we have not yet achieved the ultimate performance for waveguide-based SRS. An improved modulation scheme can increase the modulation depth from $30 \%$ to $100 \%$ and enhance the signal by 3 folds. With dedicated couplers [31], the coupling loss could be reduced from $7.5 \mathrm{~dB}$ to below $1 \mathrm{~dB}$, benefiting the SRS signal cubically (affecting both the in- and out-coupling the Stokes beam and the in -coupling of the pump beam). However, couplers with both low coupling loss and low modal reflections are not trivial to design. Therefore more work at the building block level is needed to improve the SRS enhancement factor. Adjusting the geometry of the waveguide [32] can also enhance the signal. It has been demonstrated that highly-confined slot waveguides are capable of improving the Raman conversion efficiency by 8-times. This enhancement is not yet observed in the current SRS experiments because tested slot waveguides were excessively long given their higher propagation losses. With these improvements, the SRS signal can be further enhanced by $3 \sim 4$ orders of magnitude. Considering the associated shot-noise, we expect the on-chip SRS to be capable of sub-mM detection under practical excitation. Higher sensitivity is in principle attainable in view of the noise but would be challenging to achieve in practice as it would require an extreme accuracy in background subtraction.

We report the first demonstration of SRS on a CMOScompatible silicon nitride waveguide with two CW excitations and a non-sophisticated lock-in detection scheme. Apart from the waveguide enhancement, the SRS signal is enhanced by five orders of magnitude as compared to the on-chip spontaneous result thanks to the stimulated excitation. The Raman spectra of $280 \mathrm{mM} \mathrm{(2 \% )}$ of DMSO dissolved in water have been successfully recorded from a 1-cm-long waveguide with sub-10 $\mathrm{mW}$ of excitations on the chip and a time constant of $600 \mathrm{~ms}$. Despite the extra shot noise, SRS is demonstrated to have a better performance regarding SNR than its spontaneous counterpart. With improvement on modulation, waveguide design and light coupling, we believe on-chip SRS would allow for a sub-mM detection. In replacing the deep-cooled detectors in spectrometers, our work holds the potential of enabling the full integration of the Raman sensor on a small foot-print chip compatible with manufacturing in a CMOS foundry. We envisage an on-chip multiplexed SRS sensor with integrated multi-channel lock-in amplifiers each dedicated to a specific Raman wavelength. We believe this work will open new opportunities in real-time chemical identification and quantification, constituting a versatile tool for drug development, health monitoring, and real-time environmental sensing.

\section{REFERENCES}

1. J. X. Cheng and X. S. Xie, Coherent Raman Scattering Microscopy (Taylor \& Francis, 2012).

2. C. Krafft, G. Steiner, C. Beleites, and R. Salzer, J. Biophotonics 2, 13 (2009).

3. M.-L. Xu, Y. Gao, X. X. Han, and B. Zhao, J. Agr. Food Chem. 65, 6719 (2017).

4. B. Bao, J. Riordon, F. Mostowfi, and D. Sinton, Lab Chip 17, 2740 (2017).

5. T. Steinle, V. Kumar, A. Steinmann, M. Marangoni, G. Cerullo, and H. Giessen, Opt. Lett. 40, 593 (2015).

6. G. E. Walrafen and J. Stone, Appl. Spectrosc. 26, 585 (1972).

7. S. Hanf, R. Keiner, D. Yan, J. Popp, and T. Frosch, Anal. Chem. 86, 5278 (2014)

8. S. Hanf, T. Bögözi, R. Keiner, T. Frosch, and J. Popp, Anal. Chem. 87, 982 (2015).

9. A. Khetani, V. S. Tiwari, A. Harb, and H. Anis, Opt. Express 19, 15244 (2011).

10. D. Yan, J. Popp, M. W. Pletz, and T. Frosch, ACS Photonics 4, 138 (2017).

11. W. Xie, T. Stöferle, G. Rainò, T. Aubert, S. Bisschop, Y. Zhu, R. F. Mahrt, P. Geiregat, E. Brainis, Z. Hens, and D. Van Thourhout, Adv. Mater. 29, 1604866 (2017).

12. X. Nie, E. Ryckeboer, G. Roelkens, and R. Baets, Opt. Express 25, A409 (2017)

13. A. Gondarenko, J. S. Levy, and M. Lipson, Opt. Express 17, 11366 (2009).

14. D. Dai, Z. Wang, J. F. Bauters, M.-C. Tien, M. J. R. Heck, D. J. Blumenthal, and J. E. Bowers, Opt. Express 19, 14130 (2011).

15. J. Sun, Purnawirman, E. S. Hosseini, J. D. B. Bradley, T. N. Adam, G. Leake, D. Coolbaugh, and M. R. Watts, Opt. Lett. 38, 4002 (2013).

16. A. Dhakal, A. Z. Subramanian, P. Wuytens, F. Peyskens, N. L. Thomas, and R. Baets, Opt. Lett. 39, 4025 (2014).

17. A. Dhakal, P. C. Wuytens, F. Peyskens, K. Jans, N. L. Thomas, and R. Baets, ACS Photonics 3, 2141 (2016).

18. S. A. Holmstrom, T. H. Stievater, D. A. Kozak, M. W. Pruessner, N. Tyndall, W. S. Rabinovich, R. A. McGill, and J. B. Khurgin, Optica 3, 891 (2016).

19. R. B. Miles, G. Laufer, and G. C. Bjorklund, Appl. Phys. Lett. 30, 417 (1977).

20. A. Owyoung and E. D. Jones, Opt. Lett. 1, 152 (1977).

21. C.-R. Hu, M. N. Slipchenko, P. Wang, P. Wang, J. D. Lin, G. Simpson, B. Hu, and J.-X. Cheng, Opt. Lett. 38, 1479 (2013).

22. J. L. Doménech and M. Cueto, Opt. Lett. 38, 4074 (2013).

23. P. G. Westergaard, M. Lassen, and J. C. Petersen, Opt. Express 23, 16320 (2015).

24. X. Fan and I. M. White, Nat. Photonics 5, 591 (2011).

25. K. F. Domke and B. Pettinger, J. Raman Spectrosc. 40, 1427 (2009).

26. H. Hanafusa, N. Hirose, A. Kasamatsu, T. Mimura, T. Matsui, H. M. H. Chong, H. Mizuta, and Y. Suda, Appl. Phys. Express 4, 025701 (2011).

27. R. Srnánek, J. Jakabovič, J. Kováč, J. Kováč, D. Haško, A. Šatka, E. Dobročka, and D. Donoval, Vacuum 86, 627 (2012).

28. P. C. Wuytens, H. Demol, N. Turk, K. Gevaert, A. G. Skirtach, M. Lamkanfi, and R. Baets, Faraday Discuss. (2017).

29. V. M. Wallace, N. R. Dhumal., F. M. Zehentbauer, H. J. Kim, and J. Kiefer, J. Phys. Chem. B 119, 14780 (2015).

30. P. Berto, E. R. Andresen, and H. Rigneault, Phys. Rev. Lett. 112, 053905 (2014).

31. M. Pu, L. Liu, H. Ou, K. Yvind, and J. M. Hvam, Optics Communications 283, 3678 (2010).

32. A. Dhakal, A. Raza, F. Peyskens, A. Z. Subramanian, S. Clemmen, N. L. Thomas, and R. Baets, Opt. Express 23, 27391 (2015).

\section{FUNDING INFORMATION}

ERC advanced grant InSpectra 


\section{FULL REFERENCES}

1. J. X. Cheng and X. S. Xie, Coherent Raman Scattering Microscopy (Taylor \& Francis, 2012).

2. C. Kraftt, G. Steiner, C. Beleites, and R. Salzer, "Disease recognition by infrared and raman spectroscopy," J. Biophotonics 2, 13-28 (2009).

3. M.-L. Xu, Y. Gao, X. X. Han, and B. Zhao, "Detection of pesticide residues in food using surface-enhanced raman spectroscopy: A review," J. Agr. Food Chem. 65, 6719-6726 (2017).

4. B. Bao, J. Riordon, F. Mostowfi, and D. Sinton, "Microfluidic and nanofluidic phase behaviour characterization for industrial $\mathrm{CO}_{2}$, oil and gas," Lab Chip 17, 2740-2759 (2017).

5. T. Steinle, V. Kumar, A. Steinmann, M. Marangoni, G. Cerullo, and H. Giessen, "Compact, low-noise, all-solid-state laser system for stimulated raman scattering microscopy," Opt. Lett. 40, 593-596 (2015).

6. G. E. Walrafen and J. Stone, "Intensification of spontaneous raman spectra by use of liquid core optical fibers," Appl. Spectrosc. 26, 585589 (1972).

7. S. Hanf, R. Keiner, D. Yan, J. Popp, and T. Frosch, "Fiber-enhanced raman multigas spectroscopy: A versatile tool for environmental gas sensing and breath analysis," Anal. Chem. 86, 5278-5285 (2014).

8. S. Hanf, T. Bögözi, R. Keiner, T. Frosch, and J. Popp, "Fast and highly sensitive fiber-enhanced raman spectroscopic monitoring of molecular h2 and ch4 for point-of-care diagnosis of malabsorption disorders in exhaled human breath," Anal. Chem. 87, 982-988 (2015).

9. A. Khetani, V. S. Tiwari, A. Harb, and H. Anis, "Monitoring of heparin concentration in serum by raman spectroscopy within hollow core photonic crystal fiber," Opt. Express 19, 15244-15254 (2011).

10. D. Yan, J. Popp, M. W. Pletz, and T. Frosch, "Highly sensitive broadband raman sensing of antibiotics in step-index hollow-core photonic crystal fibers," ACS Photonics 4, 138-145 (2017).

11. W. Xie, T. Stöferle, G. Rainò, T. Aubert, S. Bisschop, Y. Zhu, R. F. Mahrt, P. Geiregat, E. Brainis, Z. Hens, and D. Van Thourhout, "On-chip integrated quantum-dot-silicon-nitride microdisk lasers," Adv. Mater. 29, 1604866 (2017).

12. X. Nie, E. Ryckeboer, G. Roelkens, and R. Baets, "Cmos-compatible broadband co-propagative stationary fourier transform spectrometer integrated on a silicon nitride photonics platform," Opt. Express 25, A409-A418 (2017).

13. A. Gondarenko, J. S. Levy, and M. Lipson, "High confinement micronscale silicon nitride high q ring resonator," Opt. Express 17, 1136611370 (2009).

14. D. Dai, Z. Wang, J. F. Bauters, M.-C. Tien, M. J. R. Heck, D. J. Blumenthal, and J. E. Bowers, "Low-loss si3n4 arrayed-waveguide grating (de)multiplexer using nano-core optical waveguides," Opt. Express 19, 14130-14136 (2011).

15. J. Sun, Purnawirman, E. S. Hosseini, J. D. B. Bradley, T. N. Adam, G. Leake, D. Coolbaugh, and M. R. Watts, "Uniformly spaced $\lambda / 4-$ shifted bragg grating array with wafer-scale cmos-compatible process," Opt. Lett. 38, 4002-4004 (2013).

16. A. Dhakal, A. Z. Subramanian, P. Wuytens, F. Peyskens, N. L. Thomas, and R. Baets, "Evanescent excitation and collection of spontaneous raman spectra using silicon nitride nanophotonic waveguides," Opt. Lett. 39, 4025-4028 (2014).

17. A. Dhakal, P. C. Wuytens, F. Peyskens, K. Jans, N. L. Thomas, and R. Baets, "Nanophotonic waveguide enhanced raman spectroscopy of biological submonolayers," ACS Photonics 3, 2141-2149 (2016).

18. S. A. Holmstrom, T. H. Stievater, D. A. Kozak, M. W. Pruessner, N. Tyndall, W. S. Rabinovich, R. A. McGill, and J. B. Khurgin, "Trace gas raman spectroscopy using functionalized waveguides," Optica 3, 891896 (2016).

19. R. B. Miles, G. Laufer, and G. C. Bjorklund, "Coherent anti-stokes raman scattering in a hollow dielectric waveguide," Appl. Phys. Lett. 30, 417-419 (1977).

20. A. Owyoung and E. D. Jones, "Stimulated raman spectroscopy using low-power cw lasers," Opt. Lett. 1, 152-154 (1977).

21. C.-R. Hu, M. N. Slipchenko, P. Wang, P. Wang, J. D. Lin, G. Simpson, B. Hu, and J.-X. Cheng, "Stimulated raman scattering imaging by continuous-wave laser excitation," Opt. Lett. 38, 1479-1481 (2013).
22. J. L. Doménech and M. Cueto, "Sensitivity enhancement in high resolution stimulated raman spectroscopy of gases with hollow-core photonic crystal fibers," Opt. Lett. 38, 4074-4077 (2013).

23. P. G. Westergaard, M. Lassen, and J. C. Petersen, "Differential highresolution stimulated cw raman spectroscopy of hydrogen in a hollowcore fiber," Opt. Express 23, 16320-16328 (2015).

24. X. Fan and I. M. White, "Optofluidic microsystems for chemical and biological analysis," Nat. Photonics 5, 591-597 (2011).

25. K. F. Domke and B. Pettinger, "Tip-enhanced raman spectroscopy of $6 \mathrm{~h}$-sic with graphene adlayers: selective suppression of e1 modes," J. Raman Spectrosc. 40, 1427-1433 (2009).

26. H. Hanafusa, N. Hirose, A. Kasamatsu, T. Mimura, T. Matsui, H. M. H. Chong, H. Mizuta, and Y. Suda, "Strain distribution analysis of sputterformed strained si by tip-enhanced raman spectroscopy," Appl. Phys. Express 4, 025701 (2011).

27. R. Srnánek, J. Jakabovič, J. Kováč, J. Kováč, D. Haško, A. Šatka, E. Dobročka, and D. Donoval, "Identification of the crystalline-phases in thin pentacene layers by raman spectroscopy," Vacuum 86, 627 629 (2012).

28. P. C. Wuytens, H. Demol, N. Turk, K. Gevaert, A. G. Skirtach, M. Lamkanfi, and R. Baets, "Gold nanodome sers platform for label-free detection of protease activity," Faraday Discuss. (2017).

29. V. M. Wallace, N. R. Dhumal., F. M. Zehentbauer, H. J. Kim, and J. Kiefer, "Revisiting the aqueous solutions of dimethyl sulfoxide by spectroscopy in the mid- and near-infrared: Experiments and car-parrinello simulations," J. Phys. Chem. B 119, 14780-14789 (2015).

30. P. Berto, E. R. Andresen, and H. Rigneault, "Background-free stimulated raman spectroscopy and microscopy," Phys. Rev. Lett. 112, 053905 (2014).

31. M. Pu, L. Liu, H. Ou, K. Yvind, and J. M. Hvam, "Ultra-low-loss inverted taper coupler for silicon-on-insulator ridge waveguide," Optics Communications 283, $3678-3682$ (2010)

32. A. Dhakal, A. Raza, F. Peyskens, A. Z. Subramanian, S. Clemmen, N. L. Thomas, and R. Baets, "Efficiency of evanescent excitation and collection of spontaneous raman scattering near high index contrast channel waveguides," Opt. Express 23, 27391-27404 (2015). 